\title{
Quantum reflection from a solid surface at normal incidence
}

\author{
T. A. Pasquini, Y. Shin, C. Sanner, M. Saba, A. Schirotzek, D. E. Pritchard, W. Ketterle \\ Department of Physics, MIT-Harvard Center for Ultracold Atoms, and Research Laboratory of Electronics, \\ Massachusetts Institute of Technology, Cambridge, Massachusetts, 02139
}

(Dated: March 19, 2018)

\begin{abstract}
We observed quantum reflection of ultracold atoms from the attractive potential of a solid surface. Extremely dilute Bose-Einstein condensates of ${ }^{23} \mathrm{Na}$, with peak density $10^{11}-10^{12}$ atoms $/ \mathrm{cm}^{3}$, confined in a weak gravito-magnetic trap were normally incident on a silicon surface. Reflection probabilities of up to $20 \%$ were observed for incident velocities of $1-8 \mathrm{~mm} / \mathrm{s}$. The velocity dependence agrees qualitatively with the prediction for quantum reflection from the attractive Casimir-Polder potential. Atoms confined in a harmonic trap divided in half by a solid surface exhibited extended lifetime due to quantum reflection from the surface, implying a reflection probability above $50 \%$.
\end{abstract}

PACS numbers: 34.50.Dy, 03.75.-b, 03.75.Be

Quantum reflection is a process in which a particle reflects from a potential without reaching a classical turning point. A solid surface provides a long-range attractive potential for atoms. At separation, $r$, much larger than the atomic radius the potential follows the CasimirPolder expression $U=-C_{4} /\left(\left(r+3 \lambda / 2 \pi^{2}\right) r^{3}\right)$, where $\lambda$ is the effective atomic transition wavelength [1]. Classically, an atom incident on such a potential will be accelerated toward the surface, resulting in inelastic scattering or adsorption. A quantum mechanical treatment of an atomsurface collision reveals that the atom is reflected from the purely attractive surface potential if the potential energy changes abruptly on a length scale set by the quantum mechanical wavelength $2,3,4,4,5]$. The condition for significant reflection is that the local particle wavenumber normal to the surface, $k_{\perp}=\sqrt{k_{\infty}^{2}-2 m U / \hbar^{2}}$, change by more than $k_{\perp}$ over a distance $1 / k_{\perp}$. Here, $k_{\infty}=$ $m v_{\perp} / \hbar$ is the normal wavenumber of the atom far from the surface, $m$ is the atomic mass, $v_{\perp}$ is the normal incident velocity, and $\hbar$ is the Planck constant divided by $2 \pi$. The reflection probability, $R$, tends to unity as the incident velocity tends to zero, $R \approx 1-4 \beta_{4} m v_{\perp} / \hbar$, where $\beta_{4}$ is the length scale associated with the $C_{4}$ coefficient, $C_{4}=\beta_{4}^{2} \hbar^{2} / 2 m$. High probability quantum reflection requires low incident velocity or weak attraction to the surface, conditions previously realized only in exceptional systems.

Studies of quantum reflection were first performed with helium or hydrogen atoms incident on liquid helium surfaces [ 6, 7, 8, 9]. The extremely weak interaction strengths and low mass atoms allowed for quantum reflection at relatively high incident energies of $\sim k_{B} \times 10$ $\mathrm{mK}$ [6, 9], where $k_{B}$ is the Boltzmann constant. Reflection of noble and alkali atoms from a solid surface requires that atoms be incident with a million times less energy, $\sim k_{B} \times 10 \mathrm{nK}$. This has been accomplished only by letting untrapped atoms hit solid surfaces at grazing incidence [10, 11, 12, 13, 14], meaning that most of the velocity is directed parallel to the surface and reflection only deflects atoms slightly. Reflection probabilities in excess of $60 \%$ have been observed for incidence angles of a few milliradians 12]. Atom-surface potentials have also been studied in the presence of evanescent light waves generated by total internal reflection at a glass surface [15, 16].

Normal-incidence quantum reflection of trapped atoms may be exploited in the construction of novel atom optical devices. The current generation of atom mirrors for reflecting, confining and focusing ultracold atoms employs evanescent waves produced by total internal reflection of laser light [17] or magnetized materials [18]. An atom-optical device based on quantum reflection is in a category of its own, as it works using the universal atomsurface interaction and depends on the long wavelength of ultracold atoms. Past experiments with grazing incidence atomic beams have demonstrated a mirror [11], a reflective diffraction grating [12], and a hologram based on quantum reflection [13].

In this Letter, we demonstrate normal-incidence quantum reflection of ultracold sodium atoms. Using the harmonic trapping potential of a gravito-magnetic trap 19 , 20], we varied the center of mass velocity of dilute BoseEinstein condensates and induced controlled collisions with a silicon surface at velocities as low as $1 \mathrm{~mm} / \mathrm{s}$, corresponding to collision energies of $k_{B} \times 1.5$ nanokelvin or $1.2 \times 10^{-13} \mathrm{eV}$. A reflection probability of $\sim 20 \%$ was obtained for an incident velocity of $2 \mathrm{~mm} / \mathrm{s}$, realizing an atom mirror. Our experimental results are in qualitative agreement with theoretical predictions for single atoms incident on a conducting surface. Additionally, atoms were confined in one dimension by a silicon surface, where lifetime measurements indicate reflection probabilities in excess of $50 \%$.

Bose-Einstein condensates of ${ }^{23} \mathrm{Na}$ atoms were prepared and transferred into a gravito-magnetic trap, comprising a single coil and three external bias fields, as described in Ref. [19]. Mounted $1 \mathrm{~cm}$ above the center of the single coil was a $\sim 20 \mu \mathrm{m}$ thick N-type doped polished Si (100) surface with a resistivity of $1-10 \Omega$ $\mathrm{cm}$. For typical loading parameters, condensates con- 


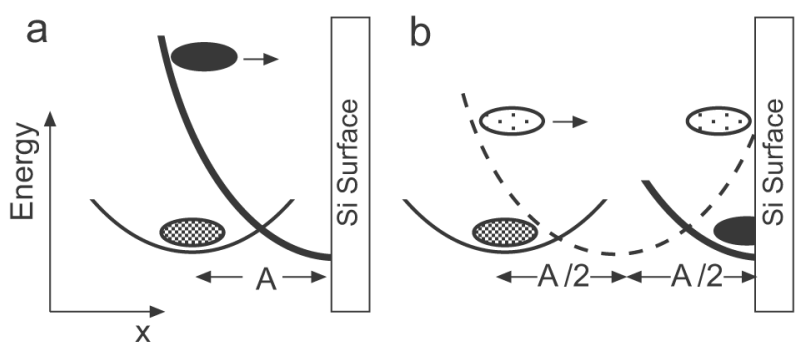

FIG. 1: Experimental schematic. Atoms were confined in a gravito-magnetic trap [19] with trap frequencies ranging from $2 \pi \times(2,2,6.5) \mathrm{Hz}$ to $2 \pi \times(11,11,6.5) \mathrm{Hz}$, near a $\sim 20 \mu \mathrm{m}$ thick Si surface. (a) Quantum reflection was studied by inducing a dipole oscillation of amplitude A perpendicular to the surface and centered on the surface. The incident velocity was varied from $1-8 \mathrm{~mm} / \mathrm{s}$ by adjusting $A$. (b) Atoms were loaded into a surface trap with zero incident velocity using an intermediate trap located at $A / 2$. Atoms initially confined at a distance $A$ from the surface were made to undergo a dipole oscillation of amplitude $A / 2$ by shifting the trap center halfway to the surface. After holding for half a trap period, $T_{\perp} / 2$, the atoms were incident on the surface with near zero velocity. The trap center was again shifted by $A / 2$ towards the surface, trapping the atoms against the $\mathrm{Si}$ wafer. To ensure contact between atoms and the surface, the center of the final trap was located $\sim 10 \%$ of the original condensate size beyond the Si surface.

taining $3 \times 10^{5}$ atoms were confined $200 \mu \mathrm{m}$ to one side of the surface in a harmonic trap characterized by $\left(\omega_{\perp}, \omega_{y}, \omega_{z}\right)=2 \pi \times(10,10,6.5) \mathrm{Hz}$, where $\omega_{\perp}$ is the horizontal trap frequency perpendicular to the surface, $\omega_{y}$ is the horizontal trap frequency parallel to the surface, and $\omega_{z}$ is the vertical trap frequency. At this point, $\omega_{\perp}$ and $\omega_{y}$ were adjusted between $11 \mathrm{~Hz}$ to $2 \mathrm{~Hz}$ by changing the vertical bias field as described in Ref. [19]. The position of the trap center relative to the surface was controlled by applying a bias field, $B_{\perp}$, perpendicular to the surface [26]. Empirically, we find that near the surface the non-condensed fraction of the atomic cloud is reduced by the "surface evaporation" effect, in which adsorption preferentially removes the hottest atoms from the cloud 21, 22].

The dipole mode of a harmonically trapped condensate is identical to the behavior of a pendulum; atoms oscillate with amplitude $A$ and trap period $T_{\perp}=2 \pi / \omega_{\perp}$. The presence of a surface within the trapping potential dramatically alters the dipole oscillation in the same way a wall would alter the oscillation of a pendulum. BoseEinstein condensates undergoing dipole oscillation in the gravito-magnetic trap were made to collide with the solid silicon surface as described in Figure 1h. Collision with the surface occurred at time $\tau_{C} \approx T_{\perp} / 4$ with incident velocity $v_{\perp}=A \omega_{\perp} \approx 1.5 \mathrm{~mm} / \mathrm{s}$. This phenomenon is observed in Figure 2 Near $\tau_{C}$, two distinct velocity classes were visible corresponding to atoms in the initial
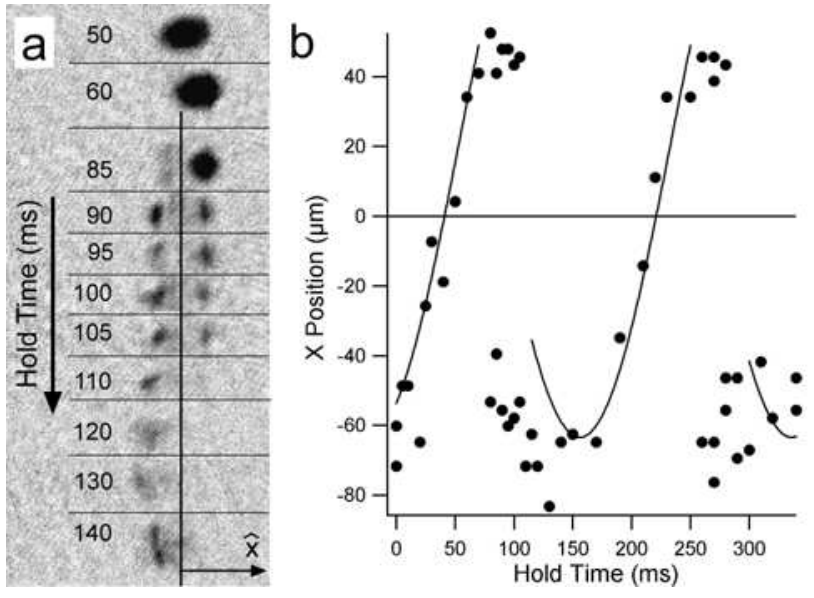

FIG. 2: Atoms reflecting from a Si surface. Atoms confined $\sim 70 \mu \mathrm{m}$ from a Si surface were transferred into a harmonic trap centered on the surface with $2 \pi \times(3.3,2.5,6.5) \mathrm{Hz}$. The dipole oscillation of the condensate was interrupted periodically by collisions with the surface, which reversed the cloud's center of mass velocity. After a variable hold time, atoms were released from the trap, fell below the edge of the surface and were imaged with resonant light after $26 \mathrm{~ms}$ time-of-flight. The position of the atoms in time-of-flight is the sum of the in-trap position at the time of release and the product of the release velocity and time-of-flight. (a) Time-of-flight images of atoms after increasing hold times show the partial transfer of atoms from the initial condensate (right) into the reflected cloud (left) as the collision occurs. The separation is due to the reversed velocity. The vertical line shows the horizontal location of the surface. The field of view is $1.4 \mathrm{~mm}$ wide. (b) The time-of-flight positions of the incident and reflected atom clouds relative to the surface are well modeled by a single particle undergoing specular reflection in a half harmonic trap (solid line). During collision, the behavior deviates from the single particle model because of the finite cloud diameter of $\sim 60 \mu \mathrm{m}$. A second reflection is visible at $270 \mathrm{~ms}$.

condensate and atoms reflected from the surface. The simultaneous presence of incident and reflected atoms is explained by the fact that the back of the condensate hits the surface $\sim 40 \mathrm{~ms}$ after the front end due to the $\sim 60$ $\mu \mathrm{m}$ condensate diameter and slow $(1.5 \mathrm{~mm} / \mathrm{s})$ velocity. At collision, the harmonic motion of the atoms was phase shifted by $2\left(\pi-\omega_{\perp} \tau_{C}\right)$, as seen in Figure $2 \mathrm{~b}$.

The reflected atom cloud was smaller than the incident one and had a comparable density. In some instances, the cloud appeared to have a bimodal distribution, indicating that coherence might be preserved in the collision. The reflected atoms continued to oscillate in the trap with the original amplitude, suggesting that atoms reflected specularly and that the kinetic energy was conserved during the collision. Eventually, the reflected atom cloud underwent a second collision with the surface at $\sim T_{\perp} / 2$ after the first collision. Additional collisions were not observed as the atom number fell below our detection limit.

We observed reflected fractions that varied from 0 - 


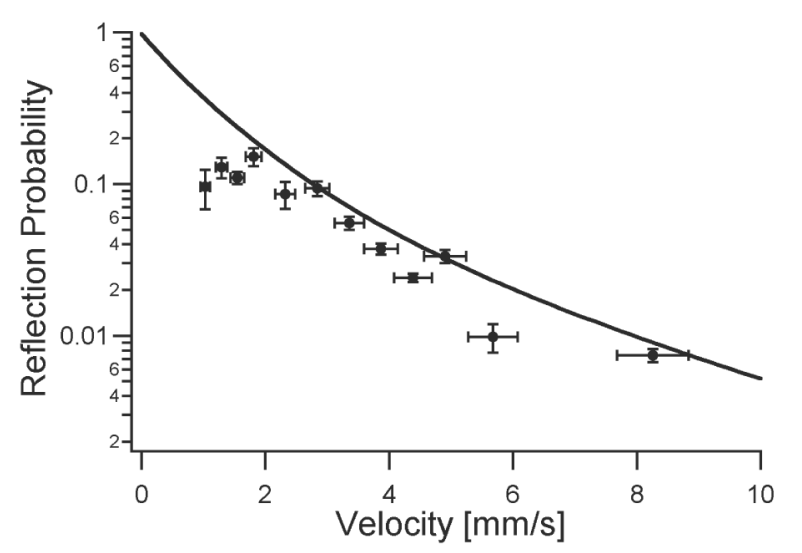

FIG. 3: Reflection probability vs incident velocity. Data were collected in a magnetic trap with trap frequencies $2 \pi \times(3.3,2.5,6.5) \mathrm{Hz}$. Incident and reflected atom numbers were averaged over several shots. Vertical error bars show the standard deviation of the mean of six measurements. Horizontal error bars reflect the uncertainty in deducing $v_{\perp}$ from the applied magnetic field $B_{\perp}$. The solid curve is a numerical calculation for individual atoms incident on a conducting surface as described in the text.

$20 \%$ over the incident velocity range of $1-8 \mathrm{~mm} / \mathrm{s}$, corresponding to a collision energy of $\sim k_{B} \times(1-100) \mathrm{nK}$, for atoms with a peak density of $\sim 2 \times 10^{12} \mathrm{~cm}^{-3}$ in a $2 \pi \times(3.3,2.5,6.5) \mathrm{Hz}$ trap. Figure 3 shows the measured reflection probability, defined as the ratio of reflected atom number to incident atom number, as a function of incident velocity. The reflection probability increases with decreasing velocity, a signature of quantum reflection. Similar behavior was observed for atoms with a peak density of $\sim 7 \times 10^{12} \mathrm{~cm}^{-3}$ in a $2 \pi \times(10.5,10,6.5) \mathrm{Hz}$ trap. For comparison, we include a line in Figure 3 showing the calculated reflection probability for a single atom incident on an conducting wall. The reflection probability was obtained by numerically solving the Schrödinger equation with the Casimir-Polder potential using the $C_{4}$ coefficient calculated for sodium atoms incident on a conducting surface, $C_{4}=9.1 \times 10^{-56} \mathrm{Jm}^{4}[1]$ and $\lambda=590$ $\mathrm{nm}$. This model ignores the harmonic trapping potential, inter-atomic forces, and electrostatic effects of adsorbed alkali atoms on the surface, which have recently been shown to distort the long range potential in the case of $\mathrm{Rb}$ atoms on insulating surfaces 23, 24]. Furthermore, the doped Si surface has a finite conductivity, leading to a reduction in $C_{4}$ of order $20-40 \%$ and a slightly higher reflection probability than a perfect conductor [25].

According to the model, reflection of atoms with 2 $\mathrm{mm} / \mathrm{s}$ velocity occurs at a distance of $\sim 1 \mu \mathrm{m}$ from the surface, where the full potential is approximated to within $10 \%$ by $U=-C_{4} / r^{4}$. The range of velocities we could explore is not large enough to investigate the region closer to the surface where the potential has a $1 / r^{3}$ de- pendence. It should be noted that without retardation, the reflection probability would be more than a hundred times lower. Ultimately, quantum reflection may be a powerful tool to characterize atom-surface interactions.

We also observe dynamics, not present in single-atom quantum reflection, when a Bose-Einstein condensate is incident on a surface. For incident velocity below 2 $\mathrm{mm} / \mathrm{s}$, the measured reflection probability remained approximately constant between 10 and $15 \%$, in contrast with theoretical predictions and previous observation. This discrepancy may be due to collective excitations of the atoms or acceleration from the harmonic trapping potential during the collision. The shape and density of the reflected atom cloud, as can be seen in Figure 2 2 , were not reproducible from shot to shot. Reflected clouds were excited and sometimes fragmented and higher velocity incident atoms tended to produce more dense reflected clouds, which may imply that an excitation occurred during the collision that was more pronounced at low collision velocities.

Furthermore, the role of the mean-field interaction energy should be considered. When a condensate is released from a trapping potential, the repulsive mean-field energy is converted into kinetic energy, imparting to the atoms an average velocity equal to the local speed of sound, $c=\sqrt{g n / m}$, where $g=4 \pi \hbar^{2} a / m$ is the coupling parameter associated with atom-atom interaction, and $a$ is the s-wave scattering length. We expect that the meanfield energy will be released during the collision so that, even for a condensate with zero center of mass velocity, the incident velocity may be characterized by the speed of sound. For Na condensates at a density of $2 \times 10^{12}$ $\mathrm{cm}^{-3}$, this velocity is $\sim 0.6 \mathrm{~mm} / \mathrm{s}$.

In the limit of zero incident velocity, a surface acting as ideal atom mirror could be used to construct a physical container for ultracold atoms and Bose-Einstein condensates. To examine the feasibility of confining atoms with solid surfaces, atoms were held in a magnetic trap divided in half by the Si surface. The transfer procedure is described schematically in Figure 1 b. Figure 4 shows the remaining fraction of atoms in the trap as a function of hold time for two different magnetic trap parameters, one at high trap frequency, $2 \pi \times(9,9,6.5)$, and the other at low trap frequency, $2 \pi \times(3.3,2.5,6.5)$. After an initial loss due to the non-zero incident velocity (not shown), the atom number was found to decrease exponentially. The lifetime for the high (low) frequency trap was $23 \mathrm{~ms}$ (170 $\mathrm{ms}$ ). We attribute the losses of atoms to scattering with the surface and adsorption. Fluctuating electro-magnetic fields in a (semi)conductor can also induce losses of atoms due to thermally induced spin flips (see, e.g., Ref. 24]). However, at the large magnetic bias field of $\sim 10 \mathrm{G}$, atoms can be ejected from the trap only with fields of frequency $\sim 7 \mathrm{MHz}$. At an average distance of $\sim 15 \mu \mathrm{m}$ from surface, the spin flip decay rate should not exceed $0.1 \mu \mathrm{Hz}$, a negligible effect in the present experiment and not a 


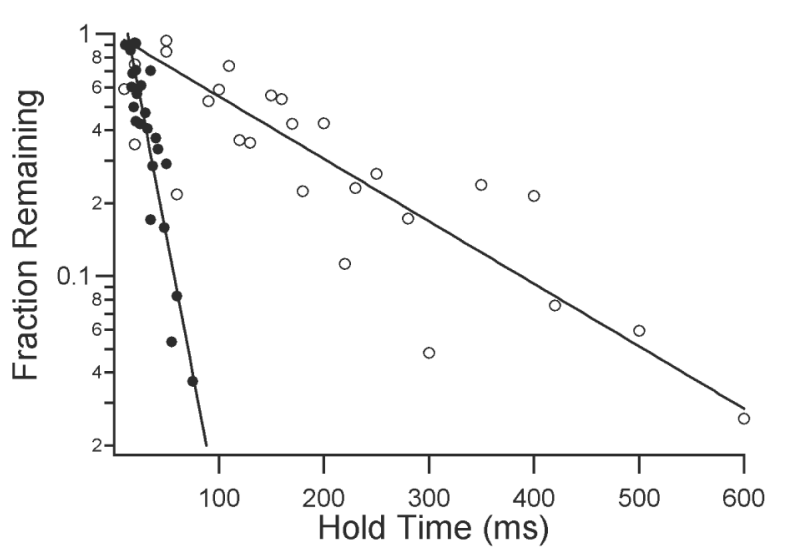

FIG. 4: Lifetime in the Si surface trap. Solid (open) circles show the remaining atom fraction vs time for a $2 \pi \times(9,9,6.5)$ $(2 \pi \times(3.3,2.5,6.5)) \mathrm{Hz}$ trapping potential with an initial atom number $3 \times 10^{4}\left(9 \times 10^{4}\right)$. The solid line exponential fit gives a lifetime of $23 \mathrm{~ms}(170 \mathrm{~ms})$ for the high (low) frequency trap geometry. The lifetime for atoms confined far from the surface exceeded $20 \mathrm{~s}$ for either geometry.

significant limitation for future ones.

In order to estimate the effect of quantum reflection on the lifetime of atoms in the trap, we assume that the atoms are incident on the surface with a velocity proportional to the speed of sound in the condensate and that the geometry of the trapped atom cloud is independent of the trap frequency. The atom loss rate to the surface may be expressed as: $d N / d t \propto-n S c(1-R)$ where $S$ is the contact area between surface and condensate. From this rate equation, we express the lifetime as $\tau_{L}=-N /(d N / d t)=\chi T_{\perp} /(1-R)$, where $\chi$ is an undetermined numerical parameter independent of the trap frequency. An identical equation would describe a thermal cloud of atoms. Comparing the ratio $\tau_{L} / T_{\perp}$ for the two different trap frequencies, we cancel out the constant $\chi$. Assuming the reflection probability for the highfrequency trap, $R_{h}=0$, gives a value of $R_{l}=60 \%$ for the low-frequency trap reflection probability. A more reasonable assumption of $R_{h}=20 \%$ gives a value of $R_{l}=70 \%$.

The results presented here demonstrate that large quantum reflection probability is not confined to exotic surfaces or extreme angles of incidence: a simple silicon wafer at room temperature can function as an atomic mirror at normal incidence, reflecting ultracold atoms. The construction of practical atom optical devices based on normal-incidence quantum reflection requires even higher reflection probabilities than demonstrated in this work. Such improvements are predicted for low-density and extremely thin surfaces [4], and have been observed with patterned surfaces, where a reduction in surface density by etching increased the maximum reflection probability by a factor of two 11]. Because reflection occurs far from the surface, uniformity of the surface is not a critical factor, as roughness is averaged over the atomic wavelength.

Surfaces are traditionally considered enemies of cold atoms: laser cooling and atom optics have developed thanks to magnetic and optical traps that confine atoms with non-material walls in ultra-high vacuum environments designed to prevent contact with surfaces. Paradoxically, it turns out that in the extreme quantum limit of nanokelvin matter waves, a surface at room temperature might become a useful device to manipulate atoms.

This work was supported by NSF, ONR, ARO, DARPA, and NASA. We thank A.E. Leanhardt for insightful discussion and J.M. Doyle for a critical reading of the manuscript. M.S. acknowledges additional support from the Swiss National Science Foundation. C.S. acknowledges the support of the Studienstiftung des deutschen Volkes.

* URL: http://cua.mit.edu/ketterle_group/

[1] H. B. G. Casimir and P. Polder, Phys. Rev. 73, 360 (1948).

[2] D. P. Clougherty and W. Kohn, Phys. Rev. B 46, 4921 (1992).

[3] C. Carraro and M. W. Cole, Prog. Surf. Sci. 57, 61 (1998).

[4] A. Mody, M. Haggerty, J. M. Doyle, and E. J. Heller, Phys. Rev. B 64, 085418 (2001).

[5] H. Friedrich, G. Jacoby, and C. G. Meister, Phys. Rev. A 65, 032902 (2002).

[6] V. U. Nayak, D. O. Edwards, and N. Masuhara, Phys. Rev. Lett. 50, 990 (1983).

[7] J. J. Berkhout et al., Phys. Rev. Lett. 63, 1689 (1989).

[8] J. M. Doyle et al., Phys. Rev. Lett. 67, 603 (1991).

[9] I. A. Yu et al., Phys. Rev. Lett. 71, 1589 (1993).

[10] A. Anderson et al., Phys. Rev. A 34, R3513 (1986).

[11] F. Shimizu, Phys. Rev. Lett. 86, 987 (2001).

[12] F. Shimizu and J. Fujita, J. Phys. Soc. Japan 71, 5 (2002).

[13] F. Shimizu and J. I. Fujita, Phys. Rev. Lett. 88, 123201 (2002).

[14] V. Druzhinina and M. DeKieviet, Phys. Rev. Lett 91, 193202 (2003).

[15] A. Landragin et al., Phys. Rev. Lett. 77, 1464 (1996).

[16] B. Segev, R. Cote, and M. G. Raizen, Phys. Rev. A 56, R3350 (1997).

[17] J. P. Dowling and J. Gea-Banacloche, Adv. At. Mol. Opt. Phys. 37, 1 (1996).

[18] E. A. Hinds and I. G. Hughes, J. Phys. D: Appl. Phys. 32, R119 (1999).

[19] A. E. Leanhardt et al., Science 301, 1513 (2003).

[20] C. R. Monroe, Ph.D. thesis, University of Colorado, Boulder (1992).

[21] O. J. Luiten et al., Phys. Rev. Lett. 70, 544 (1993).

[22] D. M. Harber, J. M. McGuirk, J. M. Obrecht, and E. A. Cornell, J. Low. Temp. Phys. 133, 229 (2003).

[23] J. M. McGuirk, D. M. Harber, J. M. Obrecht, and E. A. Cornell, Phys. Rev. A 69, 062905 (2004).

[24] Y. J. Lin, I. Teper, C. Chin, and V. Vuletic, Phys. Rev. 
Lett. 92, 050404 (2004).

[25] Z. C. Yan, A. Dalgarno, and J. F. Babb, Phys. Rev. A 55, 2882 (1997).

[26] The trap center to surface separation exhibited a linear dependance on $B_{\perp}$. The relative position of the surface was determined by loading a condensate into the trap, slowly moving the trap center toward the surface, holding for several trap periods, and measuring the remaining atom number. As the separation became smaller than the condensate radius, atoms were lost from the trap due to adsorption on the surface. The surface position was the point at which no atoms remained in the trap, and was in good agreement with the visual location of the surface determined by imaging. The position of the surface was determined to within a $10 \mu \mathrm{m}$. 